\title{
A new statistical distribution: cubic rank transmuted Kumaraswamy distribution and its properties
}

\author{
Buğra Saraçoğlu* and Caner Tanış \\ Department of Statistics, Faculty of Science, Selçuk University, Konya, Turkey.
}

\begin{abstract}
This article suggests a new statistical distribution named 'Cubic rank transmuted Kumaraswamy distribution' using cubic rank transmutation map. Various statistical properties of this new distribution such as, hazard function and its graphics, moments, variance, coefficients of skewness and kurtosis, moment generating function and order statistics are examined. In estimation problem, the maximum likelihood estimators of unknown parameters of this distribution are derived. A Monte Carlo simulation study based on bias and mean square error criteria of this estimator is conducted. Also, this new distribution is compared with other well known distributions in terms of fitting to the total milk production proportion, operation and empirical datasets in previous studies.
\end{abstract}

Keywords: Cubic rank transmutation map, cubic rank transmuted Kumaraswamy distribution, maximum likelihood estimation, Monte-Carlo simulation.

\section{INTRODUCTION}

Various statistical distributions have been widely used for modelling lifetime data in many areas such as medicine, engineering, finance and hydrology. In literature, many lifetime distributions used for statistical analysis of real datasets with decreasing, increasing and bathtube failure rates exist. Recently, many new lifetime distributions which will be used for datasets having these kind of failure rates have been obtained by using methods developed for generating new distributions in lifetime analysis. Some of the studies on this subject were conducted by Elgarhy et al. (2017), Granzotto et al. (2017), Korkmaz and Genç (2017), Celik and Guloksuz
(2017), Al-Babtain et al. (2017), Usman et al. (2017), Afify et al. (2016), Akdoğan et al. (2016), Nofal et al. (2016), Khan et al. (2016), ul Haq et al. (2016), Cordeiro et al. (2010; 2012b), Kumaraswamy (1980), Kuş (2007) etc.

The Kumaraswamy distribution proposed by Poondi Kumaraswamy (1980) has been used to model hydrological phenomena. In the area of reliability analysis, Ganji et al. (2006) have studied this distribution. In literature, many lifetime distributions which are obtained by means of this distribution exist. For example, Cordeiro and Castro (2011) have introduced family of the Kumaraswamy generalised $(\mathrm{Kw}-\mathrm{G})$ distributions. Cordeiro et al. $(2010 ; 2012 b)$ have introduced Kumaraswamy-Gumbel and KumaraswamyWeibull distributions. Kumaraswamy distribution combined with other distributions has been also used in areas such as reliability analysis, mechanical analysis as well as hydrological phenomena.

Kumaraswamy distribution with $a$ and $b$ parameters is shown by $K w(a, b)$. The cumulative distribution function (cdf) and probability density function (pdf) of this distribution are as follows;

$$
\begin{aligned}
& G(x ; a, b)=1-\left(1-x^{a}\right)^{b} \\
& g(x ; a, b)=a b x^{a-1}\left(1-x^{a}\right)^{b-1}
\end{aligned}
$$

where $a>0$ and $b>0$ are the shape parameters and $x \in[0,1]$.

\footnotetext{
* Corresponding author (bugrasarac@selcuk.edu.tr; (iD https://orcid.org/0000-0003-1713-2862)
} 
Shaw and Buckley (2009) have proposed a quadratic rank transmutation map (QRTM) to obtain new statistical distributions. This method has been used by many researchers to obtain new distributions called transmuted distributions. For example, cdf and pdf of transmuted Kumaraswamy (TKw) distribution suggested by Khan et al. (2016) are as follows;

$$
\begin{aligned}
& F(x ; a, b, \lambda)=\left[1-\lambda\left(1-x^{a}\right)^{b}\right]\left[1+\lambda\left(1-x^{a}\right)^{b}\right] \\
& f(x ; a, b, \lambda)=a b x^{a-1}\left(1-x^{a}\right)^{b-1}\left[1-\lambda+2 \lambda\left(1-x^{a}\right)^{b}\right]
\end{aligned}
$$

Afify et al. (2016) have studied the Kumaraswamy transmuted-G family of distributions. Nofal et al. (2016) have introduced a new lifetime model called Kumaraswamy transmuted exponentiated additive Weibull distribution. Al-Babtain et al. (2017) have suggested the Kumaraswamy-transmuted exponentiated modified Weibull distribution.

A dataset can fit to several statistical distributions. Therefore, it is important to obtain flexible distributions that will be used for various datasets and to determine the statistical distribution that best fits to a dataset.

The main purpose of this paper is to introduce a new distribution named cubic rank transmuted Kumaraswamy (CRTKw) distribution, which will be used to model skewed datasets and datasets having increasing and bathtube failure rates by using the cubic rank transmutation map (CRTM) proposed by Granzotto et al. (2017). The new cdf and pdf obtained with CRTM are given as follows;

$$
F(x)=\lambda_{1} G(x)+\left(\lambda_{2}-\lambda_{1}\right) G^{2}(x)+\left(1-\lambda_{2}\right) G^{3}(x)
$$

$$
f(x)=g(x)\left[\lambda_{1}+2\left(\lambda_{2}-\lambda_{1}\right) G(x)+3\left(1-\lambda_{2}\right) G^{2}(x)\right]
$$

where $G(x)$ is the cdf of any distribution and $g(x)$ is the pdf of the same distribution, $F(x)$ denotes the cdf of cubic rank transmuted G (CRT-G) distribution, $f(x)$ is the pdf of CRT-G distribution and $\lambda_{1} \in[0,1], \lambda_{2} \in[-1,1]$. Granzotto et al. (2017) have introduced cubic rank transmuted Weibull and cubic rank transmuted log-logistic distributions.

\section{METHODOLOGY}

\section{Cubic rank transmuted Kumaraswamy (CRTKw) distribution}

Let $X$ be a random variable having a CRTKw distribution with $a, b, \lambda_{1}, \lambda_{2}$ parameters. The cdf and pdf of this distribution are given in equations (7) and (8), respectively.

$$
\begin{aligned}
& F(x)=\lambda_{1}\left(1-\left(1-x^{a}\right)^{b}\right)+\left(\lambda_{2}-\lambda_{1}\right)\left(1-\left(1-x^{a}\right)^{b}\right)^{2}+ \\
& \left(1-\lambda_{2}\right)\left(1-\left(1-x^{a}\right)^{b}\right)^{3}
\end{aligned}
$$

$f(x)=a b x^{a-1}\left(1-x^{a}\right)^{b-1}\left[\lambda_{1}+2\left(\lambda_{2}-\lambda_{1}\right)\left(1-\left(1-x^{a}\right)^{b}\right)+\right.$

$\left.3\left(1-\lambda_{2}\right)\left(1-\left(1-x^{a}\right)^{b}\right)^{2}\right]$

where $a>0, b>0, \lambda_{1} \in[0,1], \lambda_{2} \in[-1,1]$ and $x \in[0,1]$. If it is taken as $\lambda_{1}=1$ and $\lambda_{2}=1$, the Kumaraswamy (Kw) distribution is obtained. The plots of pdf and cdf for various parameter values of CRTKw distribution are given in Figures 1 and 2, respectively.

The reliability function and hazard function (hf) of the CRTKw $\left(a, b, \lambda_{1}, \lambda_{2}\right)$ distribution are given in equations (9) and (10), respectively;

$$
\begin{aligned}
& \begin{aligned}
R(x) & =1-F(x) \\
& =1-\left(\lambda_{1}\left(1-\left(1-x^{a}\right)^{b}\right)+\left(\lambda_{2}-\lambda_{1}\right)\left(1-\left(1-x^{a}\right)^{b}\right)^{2}\right. \\
& \left.+\left(1-\lambda_{2}\right)\left(1-\left(1-x^{a}\right)^{b}\right)^{3}\right)
\end{aligned} \\
& h(x)=\frac{f(x)}{1-F(x)} \\
& =\frac{a b x^{a-1}\left(1-x^{a}\right)^{b-1}\left[\lambda_{1}+2\left(\lambda_{2}-\lambda_{1}\right)\left(1-\left(1-x^{a}\right)^{b}\right)+3\left(1-\lambda_{2}\right)\left(1-\left(1-x^{a}\right)^{b}\right)^{2}\right]}{1-\left(\lambda_{1}\left(1-\left(1-x^{a}\right)^{b}\right)+\left(\lambda_{2}-\lambda_{1}\right)\left(1-\left(1-x^{a}\right)^{b}\right)^{2}+\left(1-\lambda_{2}\right)\left(1-\left(1-x^{a}\right)^{b}\right)^{3}\right)}
\end{aligned}
$$

According to Figure 3, the hazard function of CRTKw $\left(a, b, \lambda_{1}, \lambda_{2}\right)$ distribution is increasing for $a>1, b>1, \lambda_{1} \in[0,1]$ and $\lambda_{2} \in[-1,1]$. On the other hand, the hazard function has a bathtub shape for $0<a<1,0<b<1, \lambda_{1} \in[0,1]$ and $\lambda_{2} \in[-1,1]$.

\section{Moments of CRTKw distribution}

Let $X$ be a random variable having a CRTKw distribution with $a, b, \lambda_{1}, \lambda_{2}$ parameters. $r^{\text {th }}$ moment of this random variable is obtained as follows; 


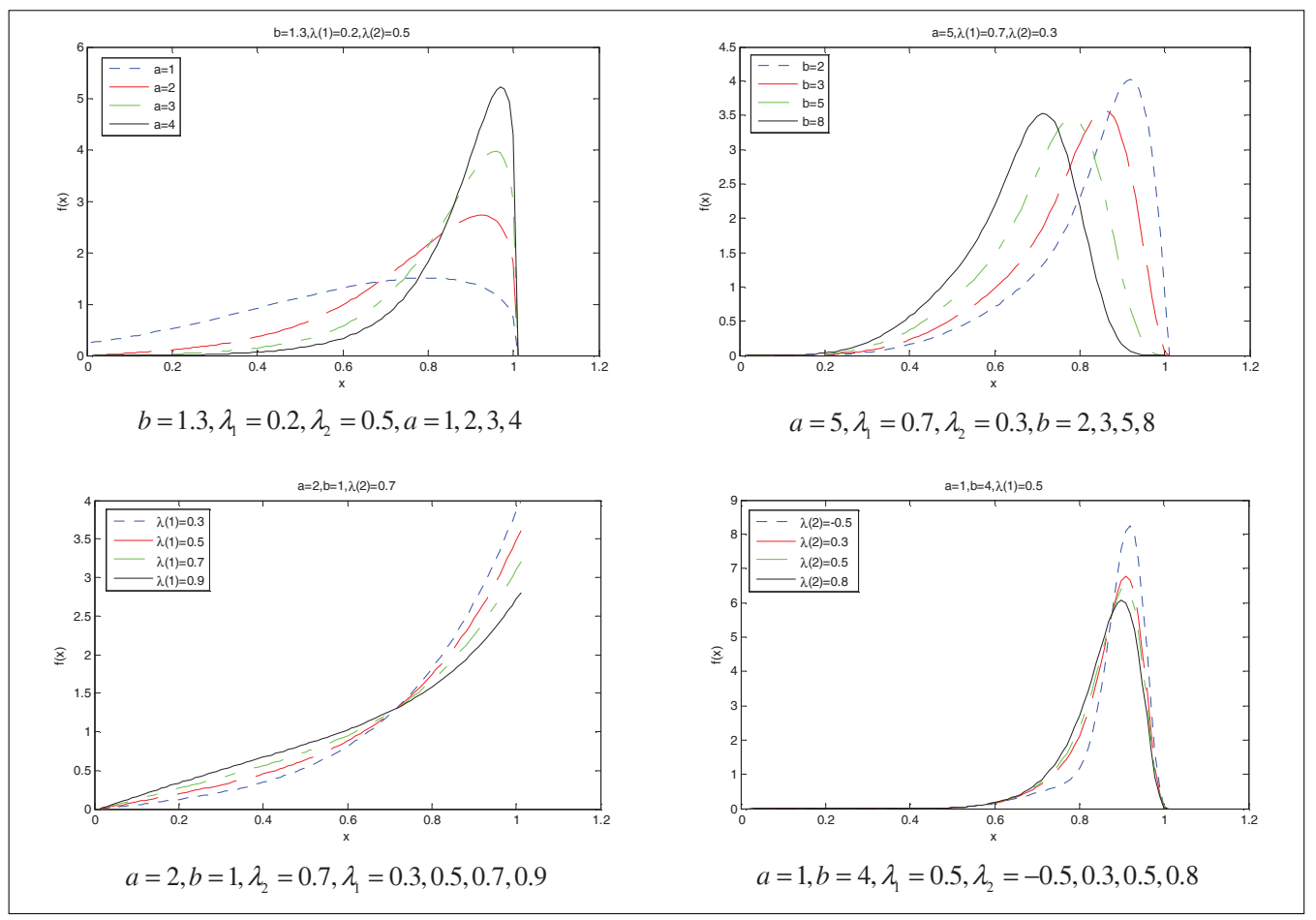

Figure 1: The plots of pdf for different parameter values of CRTKw distribution

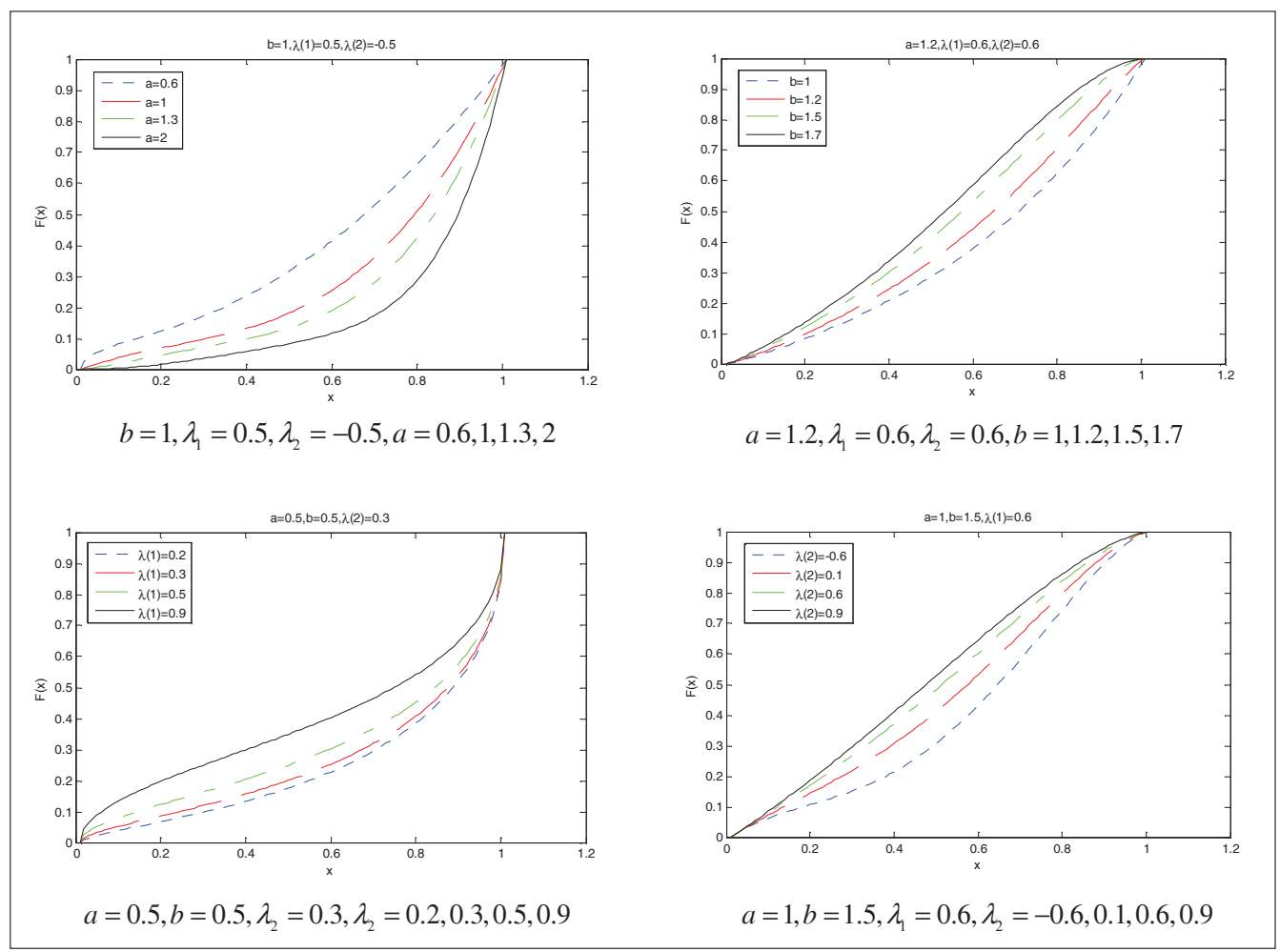

Figure 2: The plots of cdf for various parameter values for CRTKw distribution 


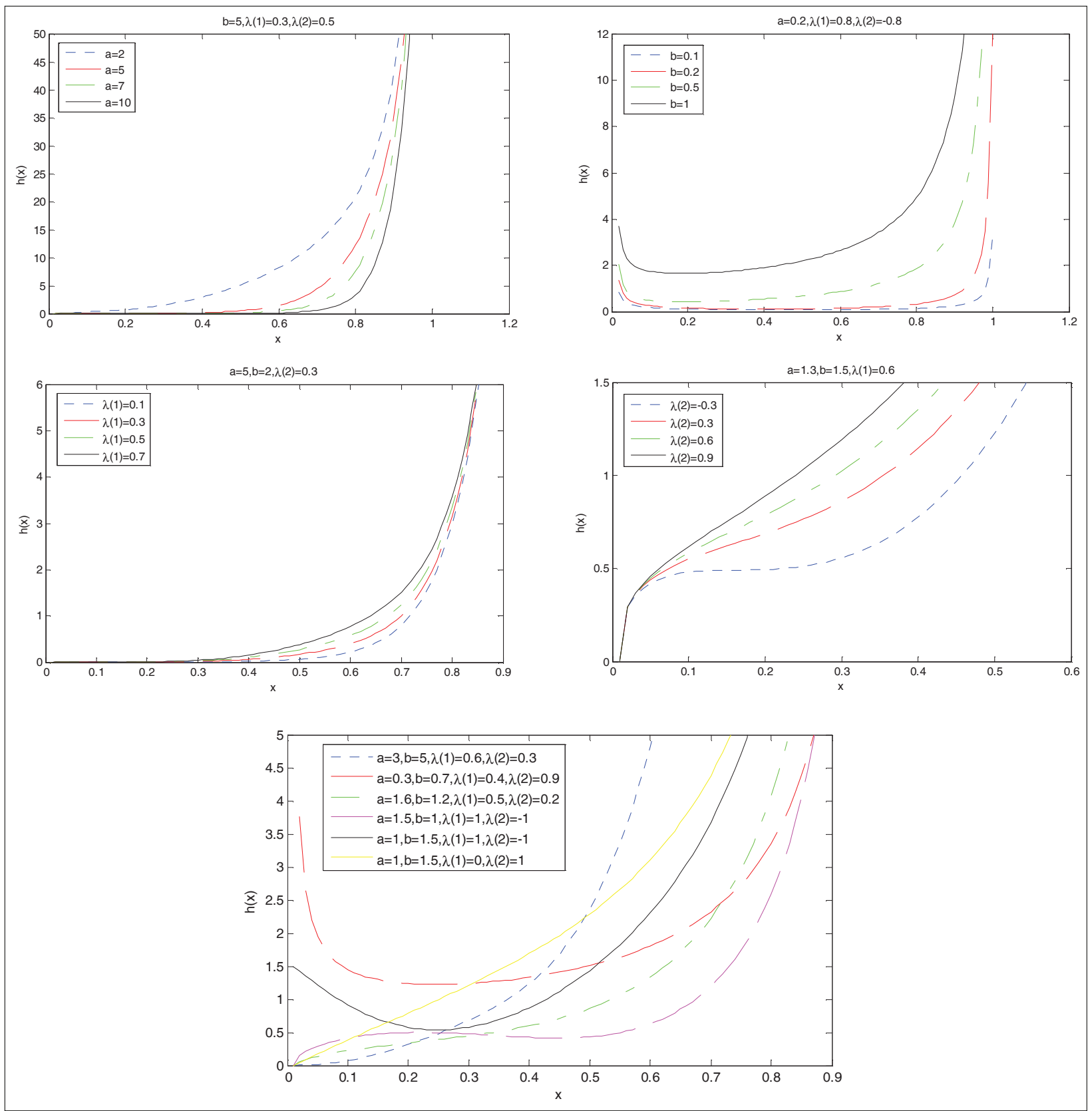

Figure 3: The plots of hf for different parameter values for CRTKw distribution

$$
\begin{aligned}
E\left(X^{r}\right)= & \int_{0}^{1} x^{r} g(x)\left[\lambda_{1}+2\left(\lambda_{2}-\lambda_{1}\right) G(x)+3\left(1-\lambda_{2}\right) G^{2}(x)\right] d x \\
= & b\left(3-\lambda_{1}-\lambda_{2}\right) \operatorname{Beta}\left(1+\frac{r}{a}, b\right)+2 b\left(2 \lambda_{2}+\lambda_{1}-3\right) \\
& \operatorname{Beta}\left(1+\frac{r}{a}, 2 b\right)+3 b\left(1-\lambda_{2}\right) \operatorname{Beta}\left(1+\frac{r}{a}, 3 b\right)
\end{aligned}
$$

where Beta (.,.) is beta function. This function is defined as follows for $\forall \alpha, \beta \in \mathfrak{R}^{+}$.

$\operatorname{Beta}(\alpha, \beta)=\int_{0}^{1} x^{\alpha-1}(1-x)^{\beta-1} d x=\frac{\Gamma(\alpha) \Gamma(\beta)}{\Gamma(\alpha+\beta)}$

where $\Gamma($.$) is gamma function and it is defined as$

$\Gamma(\alpha)=\int_{0}^{\infty} x^{\alpha-1} e^{-x} d x$

(Gradstein \& Ryzhik, 1994) 
Table 1: Mean, variance, coefficients of skewness and kurtosis for various parameter values of CRTKw distribution

\begin{tabular}{lccccc}
\hline Parameter values $\left(a, b, \lambda_{1}, \lambda_{2}\right)$ & $E(X)$ & $E\left(X^{2}\right)$ & $\operatorname{Var}(X)$ & $\mathrm{CK}$ & $\mathrm{CS}$ \\
\hline$(0.5,0.3,0.5,-0.3)$ & 0.8544 & 0.8002 & 0.0702 & 6.1734 & -2.0869 \\
$(1.3,0.7,0.5)$ & 0.3143 & 0.1427 & 0.0439 & 2.4260 & 0.4757 \\
$(3,5,0.5,-1)$ & 0.6408 & 0.4313 & 0.0206 & 5.1081 & -1.2126 \\
$(80,50,0.5,0.1)$ & 0.9525 & 0.9075 & 0.0002 & 7.3080 & -1.6164 \\
\hline
\end{tabular}

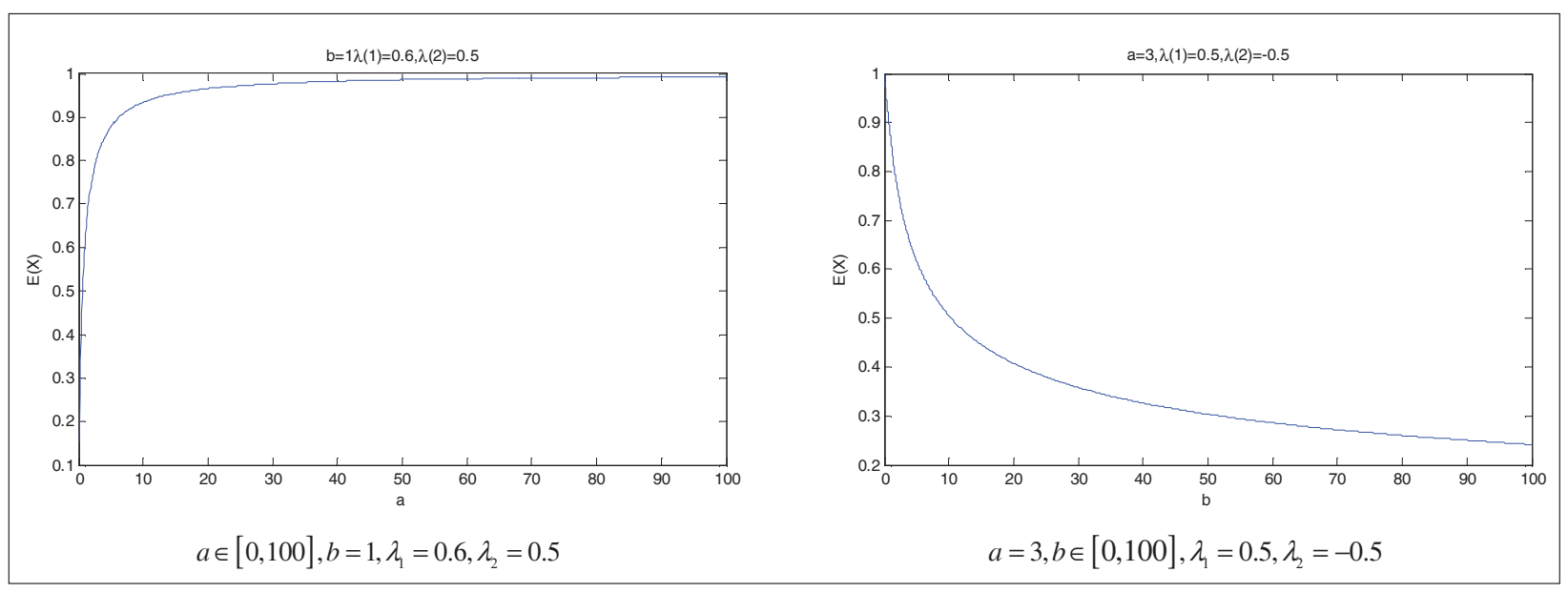

Figure 4: Expected values for different parameter values of CRTKw distribution

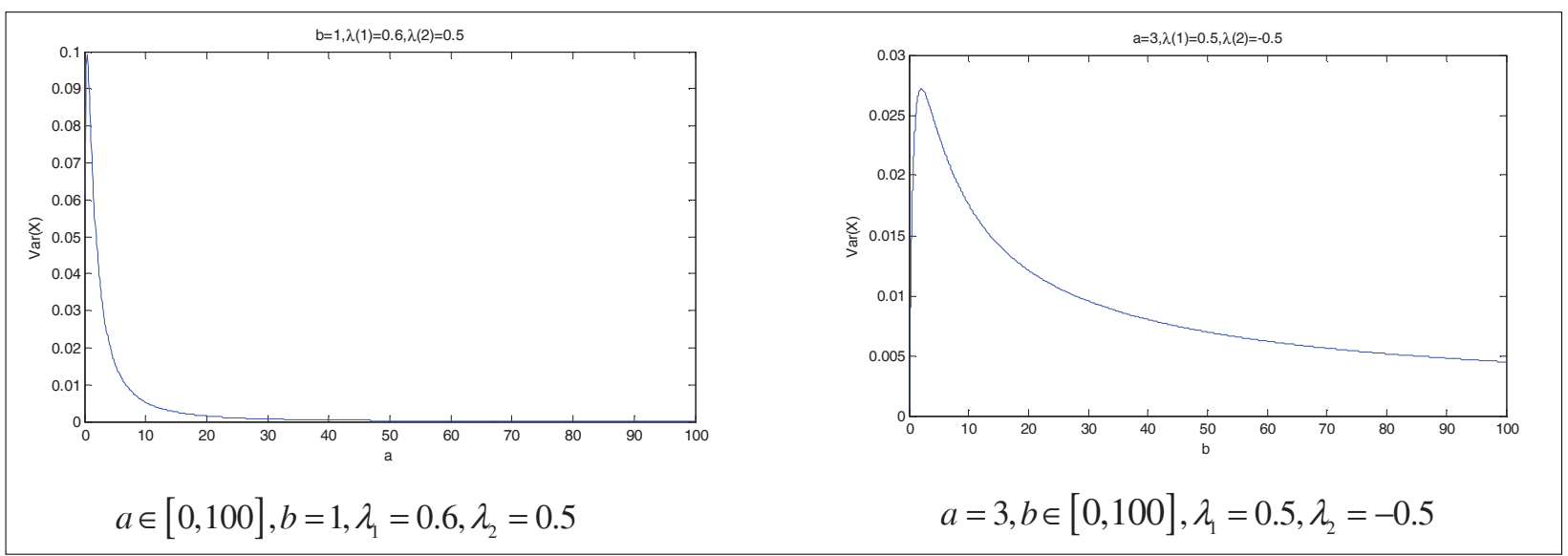

Figure 5: Variance values for different parameter values of CRTKw distribution

\section{Coefficients of skewness and kurtosis}

Coefficients of skewness and kurtosis for CRTKw distribution are given in equations (14) and (15), respectively.

$$
C S=\frac{\Gamma_{3}-3 \Gamma_{2}+2 \Gamma_{1}^{3}}{\left(\Gamma_{2}-\Gamma_{1}\right)^{\frac{3}{2}}}
$$




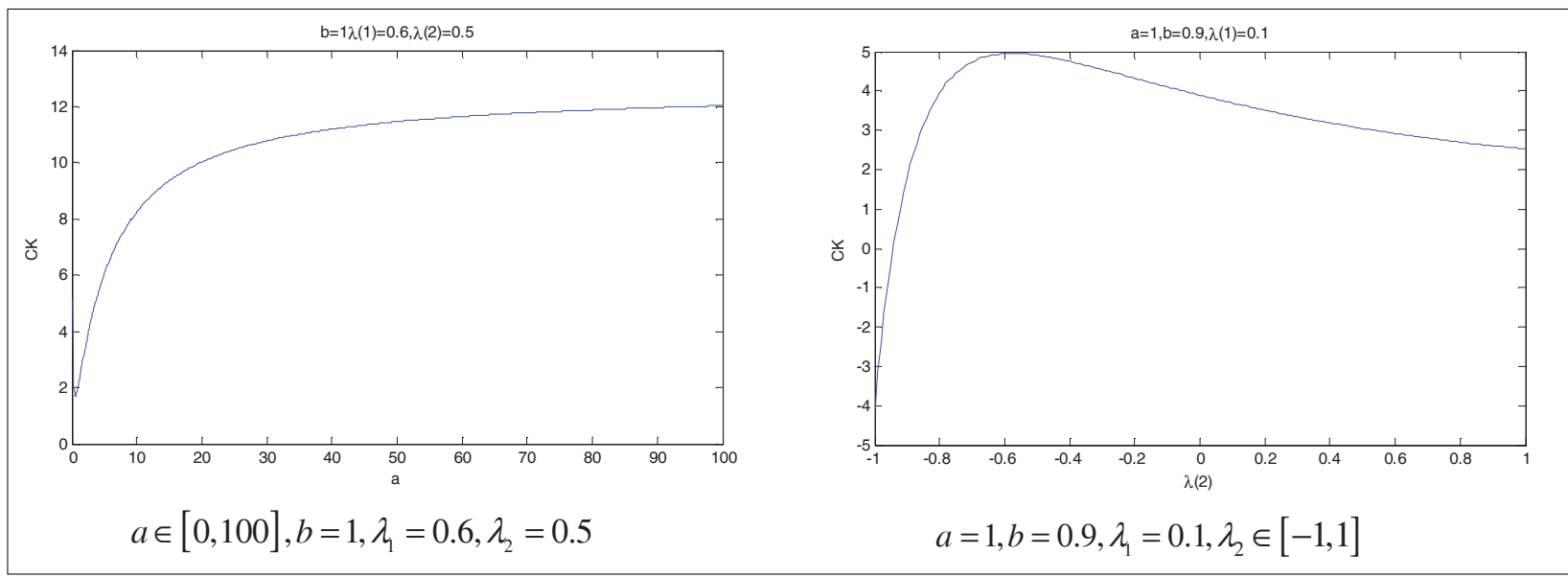

Figure 6: Coefficient of kurtosis values for different parameter values of CRTKw distribution

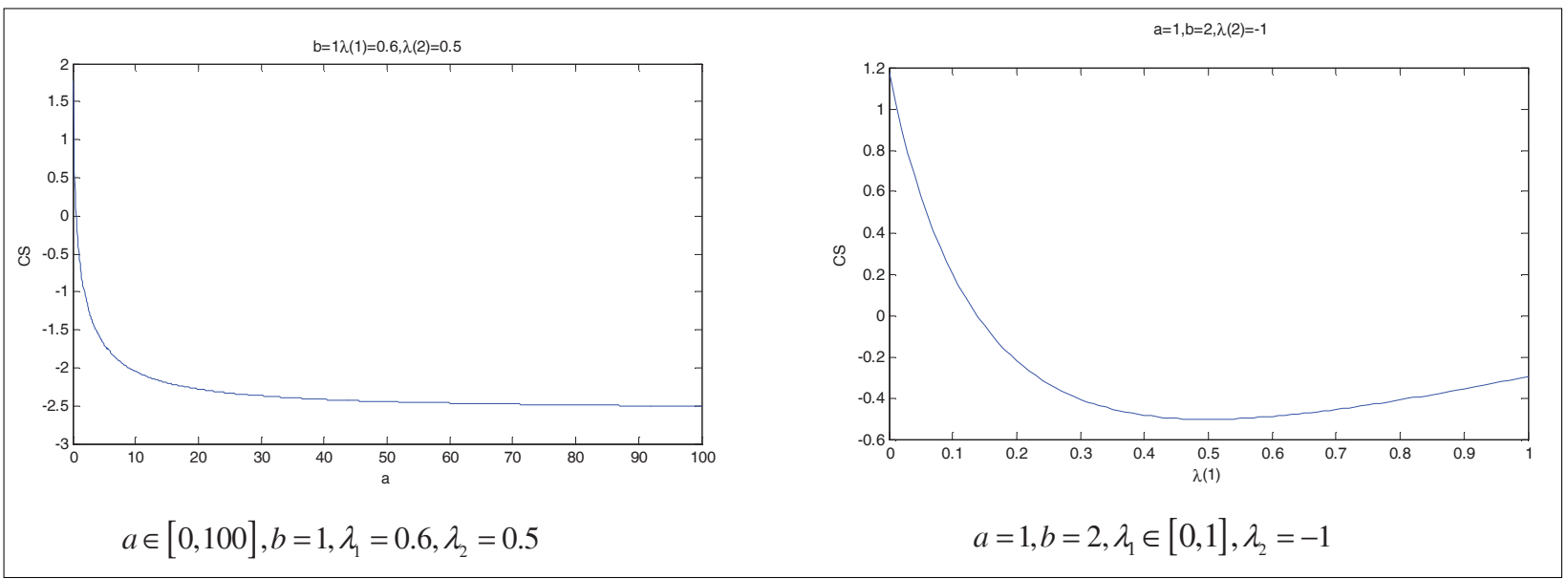

Figure 7: Coefficient of skewness values for different parameter values of CRTKw distribution

$C K=\frac{\Gamma_{4}-4 \Gamma_{1} \Gamma_{3}+6 \Gamma_{1}^{2} \Gamma_{2}-3 \Gamma_{1}^{4}}{\left[\Gamma_{2}-\Gamma_{1}^{2}\right]^{2}}$

where

$$
\begin{aligned}
\Gamma_{j}= & b\left(3-\lambda_{1}-\lambda_{2}\right) \operatorname{Bet} a\left(1+\frac{j}{a}, b\right)+ \\
& 2 b\left(2 \lambda_{2}+\lambda_{1}-3\right) \operatorname{Beta}\left(1+\frac{j}{a}, 2 b\right) \\
& +3 b\left(1-\lambda_{2}\right) \operatorname{Bet} a\left(1+\frac{j}{a}, 3 b\right), \quad j=1,2,3,4
\end{aligned}
$$

The first moment, the second moment, variance, coefficients of kurtosis and skewness for various parameter values of CRTKw distribution with $\left(a, b, \lambda_{1}, \lambda_{2}\right)$ parameters are given in Table 1.

It is seen that $a \rightarrow \infty$ then $E(X) \rightarrow b$ and $b \rightarrow \infty$ then $E(X) \rightarrow 0$. Also, as $a$ increases, variance approaches zero. It can be seen that increasing the value of $a$ parameter leads to decreasing the value of coefficient of skewness in Figure 7.

\section{Moment generating function of CRTKw distribution}

Let $X$ has a CRTKw $\left(a, b, \lambda_{1}, \lambda_{2}\right)$ distribution. Moment generating function of this distribution, $M_{x}(t)$, is obtained as follows; 


$$
\begin{aligned}
M_{x}(t)= & \int_{0}^{1} e^{t x} f(x) d x=\sum_{r=0}^{\infty} \frac{t^{r}}{r !} \int_{0}^{1} x^{r} f(x) d x \\
= & \left(3-\lambda_{1}-\lambda_{2}\right) \sum_{r=0}^{\infty} \frac{t^{r}}{r !} b \operatorname{Beta}\left(1+\frac{r}{a}, b\right)+ \\
& 2\left(2 \lambda_{2}+\lambda_{1}-3\right) \sum_{r=0}^{\infty} \frac{t^{r}}{r !} b \operatorname{Beta}\left(1+\frac{r}{a}, 2 b\right) \\
& +3\left(1-\lambda_{2}\right) \sum_{r=0}^{\infty} \frac{t^{r}}{r !} b \operatorname{Beta}\left(1+\frac{r}{a}, 3 b\right)
\end{aligned}
$$

\section{Distribution of order statistics for CRTKw distribution}

Let $X_{1}, X_{2}, \ldots, X_{n}$ be a random sample taken from CRTKw $\left(a, b, \lambda_{1}, \lambda_{2}\right)$ and $X_{(1)}, X_{(2)}, \ldots, X_{(n)}$ denote the order statistics of this random sample. In this case, the pdf of $j^{s t}$ order statistics, $f_{X_{(j)}}$, is as folllows.

$$
\begin{aligned}
f_{X_{(j)}}(x)= & A f_{X}(x)\left[F_{X}(x)\right]^{j-1}\left[1-F_{X}(x)\right]^{n-j} \\
= & A a b x^{a-1}\left(1-x^{a}\right)^{b-1}\left[\lambda_{1}+2\left(\lambda_{2}-\lambda_{1}\right)\left(1-\left(1-x^{a}\right)^{b}\right)+\right. \\
& \left.3\left(1-\lambda_{2}\right)\left(1-\left(1-x^{a}\right)^{b}\right)^{2}\right] \times\left[\lambda_{1}\left(1-\left(1-x^{a}\right)^{b}\right)+\right. \\
& \left.\left(\lambda_{2}-\lambda_{1}\right)\left(1-\left(1-x^{a}\right)^{b}\right)^{2}+\left(1-\lambda_{2}\right)\left(1-\left(1-x^{a}\right)^{b}\right)^{3}\right]^{j-1} \\
& \times\left[1-\left(\lambda_{1}\left(1-\left(1-x^{a}\right)^{b}\right)+\left(\lambda_{2}-\lambda_{1}\right)\left(1-\left(1-x^{a}\right)^{b}\right)^{2}+\right.\right. \\
& \left.\left.\left(1-\lambda_{2}\right)\left(1-\left(1-x^{a}\right)^{b}\right)^{3}\right)\right]^{n-j}
\end{aligned}
$$

where $j=1, \ldots, n$ and $A=n !((j-1) !(n-j) !)^{-1}$. The pdf of $X_{(1)}$ is given by;

$$
\begin{aligned}
f_{X_{(1)}}(x)= & n a b x^{a-1}\left(1-x^{a}\right)^{b-1}\left[\lambda_{1}+2\left(\lambda_{2}-\lambda_{1}\right)\left(1-\left(1-x^{a}\right)^{b}\right)+\right. \\
& \left.3\left(1-\lambda_{2}\right)\left(1-\left(1-x^{a}\right)^{b}\right)^{2}\right] \times\left[1-\left(\lambda_{1}\left(1-\left(1-x^{a}\right)^{b}\right)+\right.\right. \\
& \left.\left.\left(\lambda_{2}-\lambda_{1}\right)\left(1-\left(1-x^{a}\right)^{b}\right)^{2}+\left(1-\lambda_{2}\right)\left(1-\left(1-x^{a}\right)^{b}\right)^{3}\right)\right]^{n-1}
\end{aligned}
$$

The pdf of the $n^{s t}$ order statistics, $f_{X_{(n)}}(x)$, is given by

$$
\begin{aligned}
f_{X(n)}(x)= & n a b x^{a-1}\left(1-x^{a}\right)^{b-1} \times\left[\lambda_{1}+2\left(\lambda_{2}-\lambda_{1}\right)\left(1-\left(1-x^{a}\right)^{b}\right)\right. \\
& \left.+3\left(1-\lambda_{2}\right)\left(1-\left(1-x^{a}\right)^{b}\right)^{2}\right] \times\left[\lambda_{1}\left(1-\left(1-x^{a}\right)^{b}\right)\right. \\
& \left.+\left(\lambda_{2}-\lambda_{1}\right)\left(1-\left(1-x^{a}\right)^{b}\right)^{2}+\left(1-\lambda_{2}\right)\left(1-\left(1-x^{a}\right)^{b}\right)^{3}\right]^{n-1}
\end{aligned}
$$

\section{Maximum likelihood estimation for parameters of CRTKw distribution}

Let $X_{1}, X_{2}, \ldots, X_{n}$ be a random sample taken from CRTKw distribution with $\left(a, b, \lambda_{1}, \lambda_{2}\right)$ parameters. The log-likelihood function is given by;

$$
\begin{aligned}
\mid\left(a, b, \lambda_{1}, \lambda_{2} \mid \underline{x}\right)= & n(\log (a)+\operatorname{lag}(b))+(a-1) \sum_{i=1}^{n} \log \left(x_{i}\right)+ \\
& (b-1) \sum_{i=1}^{n} \log \left(1-x_{i}^{a}\right) \\
& +\sum_{i=1}^{n} \log \left[\lambda_{1}+2\left(\lambda_{2}-\lambda_{1}\right)\left(1-\left(1-x_{i}^{a}\right)^{b}\right)+\right. \\
& \left.3\left(1-\lambda_{2}\right)\left(1-\left(1-x_{i}^{a}\right)^{b}\right)^{2}\right]
\end{aligned}
$$

Differentiating the I $\left(a, b, \lambda_{1}, \lambda_{2} \mid \underline{x}\right)$ with respect to $a, b, \lambda_{1}$ and $\lambda_{2}$ parameters, and then equating to zero, the following non-linear equations are obtained;

$$
\begin{aligned}
& \frac{\partial \mathrm{l}\left(a, b, \lambda_{1}, \lambda_{2} \mid \underline{x}\right)}{\partial a}= \\
& \quad \frac{n}{a}+\sum_{i=1}^{n} \log \left(x_{i}\right)+(b-1) \sum_{i=1}^{n} \frac{x_{i}^{a} \log \left(x_{i}\right)}{x_{i}^{a}-1}-
\end{aligned}
$$

$\frac{\sum_{i=1}^{n} 2 x_{i}^{a} \log \left(x_{i}\right)\left(\left(-3 \lambda_{2}+3\right)\left(1-x_{i}^{a}\right)^{b}-3+\lambda_{1}+2 \lambda_{2}\right)\left(1-x_{i}^{a}\right)^{b} b\left(x_{i}^{a}-1\right)^{-1}}{n\left(\lambda_{1}+\lambda_{2}-3\right)-\sum_{i=1}^{n}\left(1-x_{i}^{a}\right)^{b}\left(-3 \lambda_{2}\left(1-x_{i}^{a}\right)^{b}+2 \lambda_{1}+4 \lambda_{2}+3\left(1-x_{i}^{a}\right)^{b}-6\right)}=0$

$\frac{\partial \mathrm{l}\left(a, b, \lambda_{1}, \lambda_{2} \mid \underline{x}\right)}{\partial b}=\frac{n}{b}+\sum_{i=1}^{n} \log \left(1-x_{i}^{a}\right)-$

$\frac{\sum_{i=1}^{n}\left[2\left(1-x_{i}^{a}\right)^{b} \log \left(1-x_{i}^{a}\right)\left(\left(-3 \lambda_{2}+3\right)\left(1-x_{i}^{a}\right)^{b}-3+\lambda_{1}+2 \lambda_{2}\right)\right]}{n\left(\lambda_{1}+\lambda_{2}-3\right)-\sum_{i=1}^{n}\left(1-x_{i}^{a}\right)^{b}\left(-3 \lambda_{2}\left(1-x_{i}^{a}\right)^{b}+2 \lambda_{1}+4 \lambda_{2}+3\left(1-x_{i}^{a}\right)^{b}-6\right)}=0$ 
$\frac{\partial \mathrm{l}\left(a, b, \lambda_{1}, \lambda_{2} \mid \underline{x}\right)}{\partial \lambda_{1}}=$

$=\frac{n-2 \sum_{i=1}^{n}\left(1-x_{i}^{a}\right)^{b}}{n\left(\lambda_{1}+\lambda_{2}-3\right)-\sum_{i=1}^{n}\left(1-x_{i}^{a}\right)^{b}\left(-3 \lambda_{2}\left(1-x_{i}^{a}\right)^{b}+2 \lambda_{1}+4 \lambda_{2}+3\left(1-x_{i}^{a}\right)^{b}-6\right)}=0$

$\underline{\ell\left(a, b, \lambda_{1}, \lambda_{2} \mid \underline{x}\right)}=$

$\partial \lambda_{2}$

$=\frac{n-\sum_{i=1}^{n} 4\left(1-x_{i}^{a}\right)^{b}-3\left(1-x_{i}^{a}\right)^{2 b}}{n\left(\lambda_{1}+\lambda_{2}-3\right)-\sum_{i=1}^{n}\left(1-x_{i}^{a}\right)^{b}\left(-3 \lambda_{2}\left(1-x_{i}^{a}\right)^{b}+2 \lambda_{1}+4 \lambda_{2}+3\left(1-x_{i}^{a}\right)^{b}-6\right)}=0$

These non-linear equations can be solved by using the Newton-Raphson method. Finally, MLEs for $a, b, \lambda_{1}$ and $\lambda_{2}$ parameters are obtained.

\section{RESULTS AND DISCUSSION}

\section{Simulation study}

In this section, a Monte-Carlo simulation study is given to indicate the performances of MLEs of unknown parameters of CRTKW $\left(a, b, \lambda_{1}, \lambda_{2}\right)$ distribution for different parameter values and different sample sizes. The biases and MSEs of the MLEs are simulated based on 5000 replicates for different sample sizes such as 50 , 100, 200, 300, 500, 1000 and for different parameter values such as $(0.4,0.3,0.3,0.8),(0.5,1.3,0.1,0.7)$, $(1,0.5,0.2,0.9),(1,1.2,0.2,0.9)$.

The simulation results are given in Table 2. According to Table 2, as sample sizes increase, the MSEs and biases of MLEs decrease for all parameter values. This situation indicates that estimated value approaches real value as sample size increases.

Table 2: Biases and MSEs of MLEs for various values of parameters

\begin{tabular}{|c|c|c|c|c|c|c|c|c|c|}
\hline \multirow[b]{2}{*}{$\mathrm{n}$} & \multirow[b]{2}{*}{ parameters } & \multicolumn{2}{|c|}{$\hat{a}$} & \multicolumn{2}{|c|}{$\hat{b}$} & \multicolumn{2}{|c|}{$\hat{\lambda}_{1}$} & \multicolumn{2}{|c|}{$\hat{\lambda}_{2}$} \\
\hline & & bias & mse & bias & mse & bias & mse & bias & mse \\
\hline 50 & \multirow{6}{*}{$(0.4,0.3,0.3,0.8)$} & 0.1176 & 0.0804 & 0.0460 & 0.0052 & 0.2348 & 0.1158 & -0.8092 & 0.9020 \\
\hline 100 & & 0.0970 & 0.0537 & 0.0317 & 0.0027 & 0.2050 & 0.1079 & -0.6096 & 0.5637 \\
\hline 200 & & 0.0721 & 0.0380 & 0.0197 & 0.0012 & 0.1569 & 0.0840 & -0.4253 & 0.3114 \\
\hline 300 & & 0.0570 & 0.0296 & 0.0144 & 0.0008 & 0.1248 & 0.0672 & -0.3302 & 0.2055 \\
\hline 500 & & 0.0401 & 0.0199 & 0.0104 & 0.0005 & 0.0873 & 0.0473 & -0.2350 & 0.1239 \\
\hline 1000 & & 0.0185 & 0.0113 & 0.0056 & 0.0002 & 0.0450 & 0.0269 & -0.1327 & 0.0563 \\
\hline 50 & \multirow{6}{*}{$(0.5,1.3,0.1,0.7)$} & 0.1156 & 0.0566 & 0.2832 & 0.1910 & 0.3691 & 0.1955 & -0.8333 & 0.9154 \\
\hline 100 & & 0.0850 & 0.0430 & 0.1821 & 0.0853 & 0.2960 & 0.1502 & -0.6399 & 0.6246 \\
\hline 200 & & 0.0596 & 0.0371 & 0.1034 & 0.0373 & 0.2290 & 0.1161 & -0.4710 & 0.4149 \\
\hline 300 & & 0.0512 & 0.0323 & 0.0794 & 0.0260 & 0.1908 & 0.0948 & -0.3698 & 0.3045 \\
\hline 500 & & 0.0301 & 0.0241 & 0.0446 & 0.0139 & 0.1339 & 0.0663 & -0.2778 & 0.2206 \\
\hline 1000 & & 0.0154 & 0.0174 & 0.0222 & 0.0065 & 0.0882 & 0.0436 & -0.1989 & 0.1508 \\
\hline 50 & \multirow{6}{*}{$(1,0.5,0.2,0.9)$} & 0.2591 & 0.3677 & 0.0921 & 0.0186 & 0.3109 & 0.1574 & -0.9528 & 1.1511 \\
\hline 100 & & 0.1824 & 0.2581 & 0.0649 & 0.0090 & 0.2509 & 0.1270 & -0.7629 & 0.7875 \\
\hline 200 & & 0.1415 & 0.2077 & 0.0430 & 0.0043 & 0.1990 & 0.1015 & -0.5790 & 0.4906 \\
\hline 300 & & 0.1146 & 0.1735 & 0.0338 & 0.0028 & 0.1670 & 0.0862 & -0.4763 & 0.3614 \\
\hline 500 & & 0.0607 & 0.1389 & 0.0230 & 0.0016 & 0.1141 & 0.0620 & -0.3756 & 0.2523 \\
\hline 1000 & & 0.0307 & 0.0995 & 0.0143 & 0.0007 & 0.0727 & 0.0410 & -0.2618 & 0.1486 \\
\hline 50 & \multirow{6}{*}{$(1,1.2,0.2,0.9)$} & 0.1327 & 0.1604 & 0.2473 & 0.1468 & 0.3169 & 0.1588 & -0.9840 & 1.2150 \\
\hline 100 & & 0.0835 & 0.1289 & 0.1633 & 0.0695 & 0.2529 & 0.1297 & -0.8019 & 0.8636 \\
\hline 200 & & 0.0636 & 0.1133 & 0.1049 & 0.0332 & 0.2004 & 0.1109 & -0.5992 & 0.5289 \\
\hline 300 & & 0.0445 & 0.0990 & 0.0769 & 0.0210 & 0.1652 & 0.0963 & -0.4987 & 0.3960 \\
\hline 500 & & 0.0248 & 0.0844 & 0.0526 & 0.0122 & 0.1258 & 0.0788 & -0.4032 & 0.2790 \\
\hline 1000 & & -0.0116 & 0.0615 & 0.0282 & 0.0055 & 0.0679 & 0.0520 & -0.3123 & 0.1864 \\
\hline
\end{tabular}




\section{Real data analysis}

In this section, two real data applications are presented to illustrate the applicability of CRTKw distribution in real life. The first application is with regard to the data about the total milk production proportion in the first birth of 107 cows living in the Carnaúba farm in Brazil. These data exist in studies of Cordeiro and Brito (2012a) and Brito (2009) and it has also been used by Yousof et al. (2017). The dataset is as follows;

\section{Milk production data}

\begin{tabular}{lllllllllll}
\hline 0.4365 & 0.4260 & 0.5140 & 0.6907 & 0.7471 & 0.2605 & 0.6196 & 0.8781 & 0.4990 & 0.6058 & 0.6891 \\
0.5770 & 0.5394 & 0.1479 & 0.2356 & 0.6012 & 0.1525 & 0.5483 & 0.6927 & 0.7261 & 0.3323 & 0.0671 \\
0.2361 & 0.4800 & 0.5707 & 0.7131 & 0.5853 & 0.6768 & 0.5350 & 0.4151 & 0.6789 & 0.4576 & 0.3259 \\
0.2303 & 0.7687 & 0.4371 & 0.3383 & 0.6114 & 0.3480 & 0.4564 & 0.7804 & 0.3406 & 0.4823 & 0.5912 \\
0.5744 & 0.5481 & 0.1131 & 0.7290 & 0.0168 & 0.5529 & 0.4530 & 0.3891 & 0.4752 & 0.3134 & 0.3175 \\
0.1167 & 0.6750 & 0.5113 & 0.5447 & 0.4143 & 0.5627 & 0.5150 & 0.0776 & 0.3945 & 0.4553 & 0.4470 \\
0.5285 & 0.5232 & 0.6465 & 0.0650 & 0.8492 & 0.8147 & 0.3627 & 0.3906 & 0.4438 & 0.4612 & 0.3188 \\
0.2160 & 0.6707 & 0.6220 & 0.5629 & 0.4675 & 0.6844 & 0.3413 & 0.4332 & 0.0854 & 0.3821 & 0.4694 \\
0.3635 & 0.4111 & 0.5349 & 0.3751 & 0.1546 & 0.4517 & 0.2681 & 0.4049 & 0.5553 & 0.5878 & 0.4741 \\
0.3598 & 0.7629 & 0.5941 & 0.6174 & 0.6860 & 0.0609 & 0.6488 & 0.2747 & & & \\
\hline
\end{tabular}

These data are fitted to CRTKw, Kumaraswamy (Kw), transmuted Kumaraswamy (TKw), exponentiated Kumararaswamy (EKw), Weibull and transmuted exponentiated exponential (TEE) distributions. In order to show whether these distributions fit to the dataset, measures such as, the Kolmogorov-Smirnov (KS) statistics, Anderson-Darling goodness-of-fit statistics $\left(A^{*}\right)$, Akaike's information criterion (AIC), corrected Akaike's information criterion (AICc), the Bayesian information criterion (BIC) and $-2 \times \log$-likelihood value have been computed. These measures are given by;

$$
A I C=-2 \ell+2 k
$$

$$
A I C c=A I C+\left(\frac{2 k(k+1)}{n-k-1}\right)
$$

$$
\begin{aligned}
& B I C=-2 \ell+k \log (n) \\
& A^{*}=-n-\frac{1}{n} \sum_{j=1}^{n}(2 j-1) \log \left[F\left(X_{(j)}\right)\left(1-F\left(X_{(n-j+1)}\right)\right)\right]
\end{aligned}
$$

$$
K S=\sup \left(\left|F(x)-F_{n}(x)\right|\right)
$$

where $X_{(j)}$ is $\mathrm{j}^{\text {st }}$ order statistics, $k$ is the number of parameters and $\mathrm{n}$ is the sample size. $\ell$ is the value of log-likelihood function and $F_{n}(x)$ is the empirical distribution function.

The density function of the Kw, TKw, EKw, Weibull and TEE distributions are given by;

$$
\begin{gathered}
T K w: f(x)=a b x^{a-1}\left(1-x^{a}\right)^{b-1}\left[1-\lambda+2 \lambda\left(1-x^{a}\right)^{b}\right] \\
a, b>0, x \in[0,1], \lambda \in[-1,1]
\end{gathered}
$$

$$
\begin{gathered}
E K w: f(x)=\theta a b x^{a-1}\left(1-x^{a}\right)^{b-1}\left[1-\left(1-x^{a}\right)^{b}\right]^{\theta-1} \\
a, b, \theta>0, x \in[0,1]
\end{gathered}
$$

$K w: f(x)=a b x^{a-1}\left(1-x^{a}\right)^{b-1} \quad a, b>0, x \in[0,1]$

Weibull : $f(x)=\frac{\mu}{\sigma}\left(\frac{x}{\sigma}\right)^{\mu-1} e^{-\left(\frac{x}{\sigma}\right)^{\mu}} x, \mu, \sigma>0$

$$
\begin{gathered}
\text { TEE : } f(x)=\theta \alpha\left(1-e^{-\alpha x}\right)^{\theta-1} e^{-\alpha x}\left[1+\lambda-2 \lambda\left(1-e^{-\alpha x}\right)^{\theta}\right] \\
\alpha, \theta, x>0, \lambda \in[-1,1]
\end{gathered}
$$

ML estimates for unknown parameters of these distributions and their standard errors are given in Tables 3 and 4. Plots of the empirical and theoretical cdfs and pdfs for fitted distributions are given in Figures 8 and 9, respectively. 
Table 3: Parameter estimate (standard errors) for milk production dataset

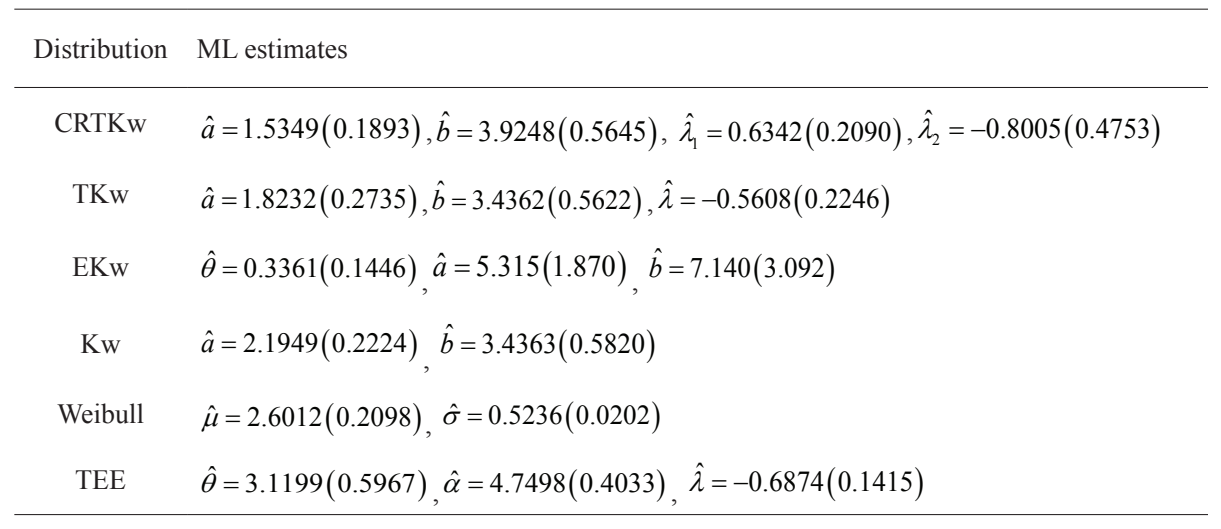

Table 4: Selection criteria statistics for milk production data

\begin{tabular}{lrrrrrrr}
\hline Distribution & $-2 \log$ & AIC & AICc & BIC & A* & K-S & p value \\
\hline CRTKw & $\mathbf{- 6 2 . 2 2 3}$ & $\mathbf{- 5 4 . 2 2 3}$ & $\mathbf{- 5 3 . 8 3 1}$ & $\mathbf{- 4 3 . 5 3 2}$ & $\mathbf{0 . 1 1 7 6}$ & $\mathbf{0 . 0 4 2 7}$ & $\mathbf{0 . 9 8 9 9}$ \\
TKw & -54.097 & -48.097 & -47.864 & -40.079 & 0.6361 & 0.0600 & 0.8357 \\
EKw & -27.557 & -49.114 & -48.881 & -41.443 & 0.5950 & 0.0748 & 0.5868 \\
Kw & -50.789 & -46.789 & -46.674 & -41.443 & 1.0031 & 0.0763 & 0.5625 \\
Weibull & -42.695 & -38.695 & -38.579 & -33.349 & 1.4839 & 0.0832 & 0.4488 \\
TEE & -20.176 & -14.176 & -13.943 & -6.157 & 3.4382 & 0.1232 & 0.0777 \\
\hline
\end{tabular}

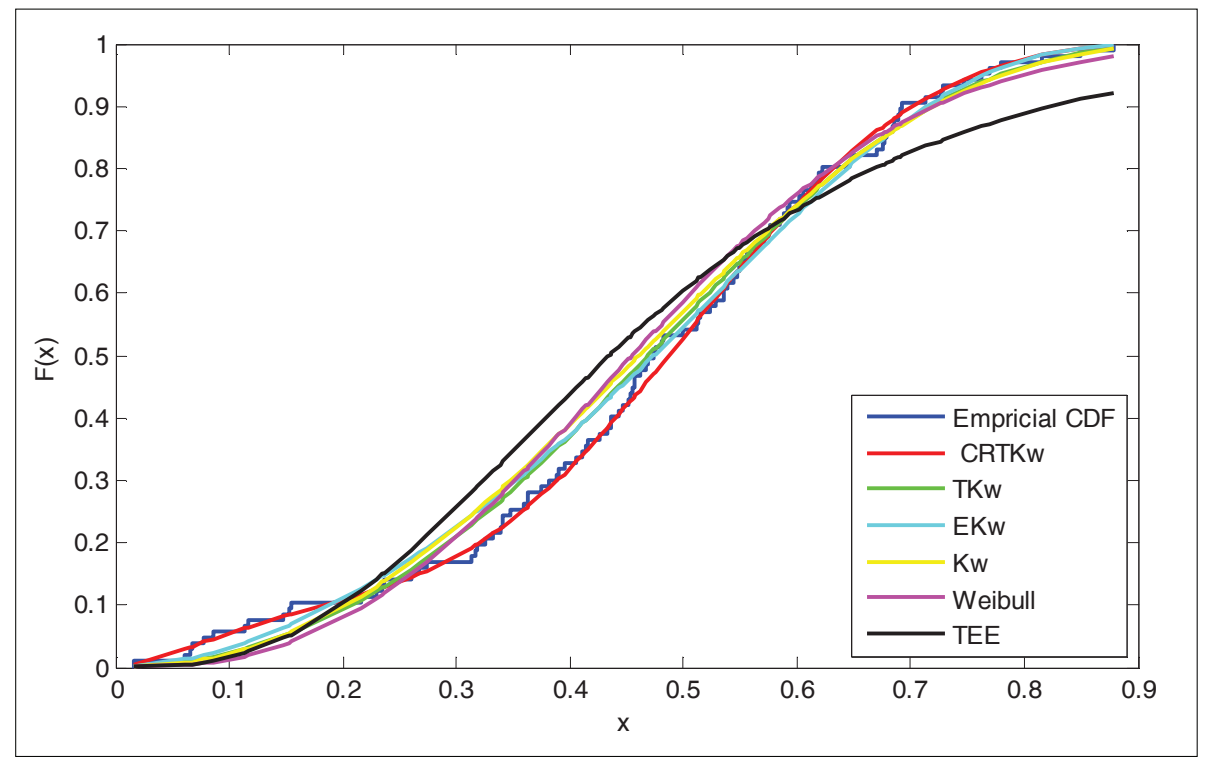

Figure 8: Empirical cdf and theoretical cdf for milk production dataset 


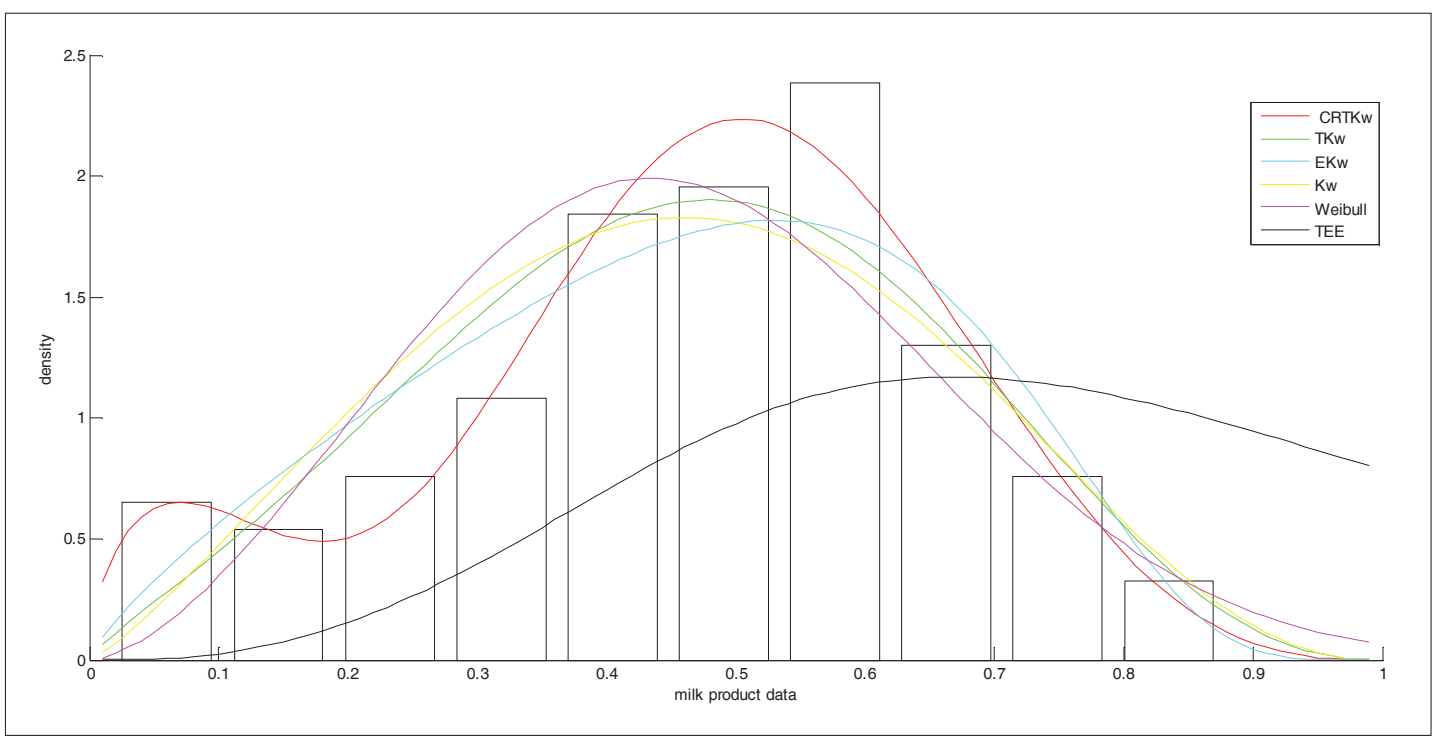

Figure 9: Fitted pdf for milk production dataset

As seen from the above plots, the best fit for this dataset is the CRTKw distribution. It can be suggested that CRTKw distribution should be used for this dataset.

\section{Operation and empirical data}

The second real dataset, which consists of 50 observations has been obtained by Dasgupta (2011). This dataset is related to holes operation on jobs made of iron sheet. This dataset is as follows;

\begin{tabular}{llllllllll}
\hline 0.04 & 0.02 & 0.06 & 0.12 & 0.14 & 0.08 & 0.22 & 0.12 & 0.08 & 0.26 \\
0.24 & 0.04 & 0.14 & 0.16 & 0.08 & 0.26 & 0.32 & 0.28 & 0.14 & 0.16 \\
0.24 & 0.22 & 0.12 & 0.18 & 0.24 & 0.32 & 0.16 & 0.14 & 0.08 & 0.16 \\
0.24 & 0.16 & 0.32 & 0.18 & 0.24 & 0.22 & 0.16 & 0.12 & 0.24 & 0.06 \\
0.02 & 0.18 & 0.22 & 0.14 & 0.06 & 0.04 & 0.14 & 0.26 & 0.18 & 0.16 \\
\hline
\end{tabular}

This dataset has been fitted to CRTKw, Kw, TKw, transmuted Rayleigh (TR), exponentiated exponential (EE), Weibull and transmuted Weibull (TW) distributions. The pdf of the fitted distributions are given by;

$$
\begin{gathered}
T K w: f(x)=a b x^{a-1}\left(1-x^{a}\right)^{b-1}\left[1-\lambda+2 \lambda\left(1-x^{a}\right)^{b}\right] \\
a, b>0, x \in[0,1], \lambda \in[-1,1]
\end{gathered}
$$

$T R: f(x)=\frac{x}{\sigma^{2}} e^{-\frac{x^{2}}{2 \sigma^{2}}}\left[1-\lambda+2 \lambda e^{-\frac{x^{2}}{2 \sigma^{2}}}\right] \quad \sigma, x>0$

$E E: f(x)=\theta \alpha\left(1-e^{-\alpha x}\right)^{\theta-1} e^{-\alpha x} \quad \alpha, \theta, x>0$

$K w: f(x)=a b x^{a-1}\left(1-x^{a}\right)^{b-1} \quad a, b>0, x \in[0,1]$

Weibull : $f(x)=\frac{\mu}{\sigma}\left(\frac{x}{\sigma}\right)^{\mu-1} e^{-\left(\frac{x}{\sigma}\right)^{\mu}} x, \mu, \sigma>0$

$\mathrm{TW}: f(x)=\frac{\mu}{\sigma}\left(\frac{x}{\sigma}\right)^{\mu-1} e^{-\left(\frac{x}{\sigma}\right)^{\mu}}\left[1-\lambda+2 \lambda e^{-\left(\frac{x}{\sigma}\right)^{\mu}}\right]$

$$
x, \mu, \sigma>0, \lambda \in[-1,1]
$$

ML estimates for unknown parameters of these distributions and their standard errors are given in Tables 5 and 6. Further, plots of the empirical cdf, theoretical cdfs and pdfs for fitted distributions are given in Figure 10 and Figure 11, respectively. 
Table 5: Parameter estimates (standard errors) for operation and empirical dataset

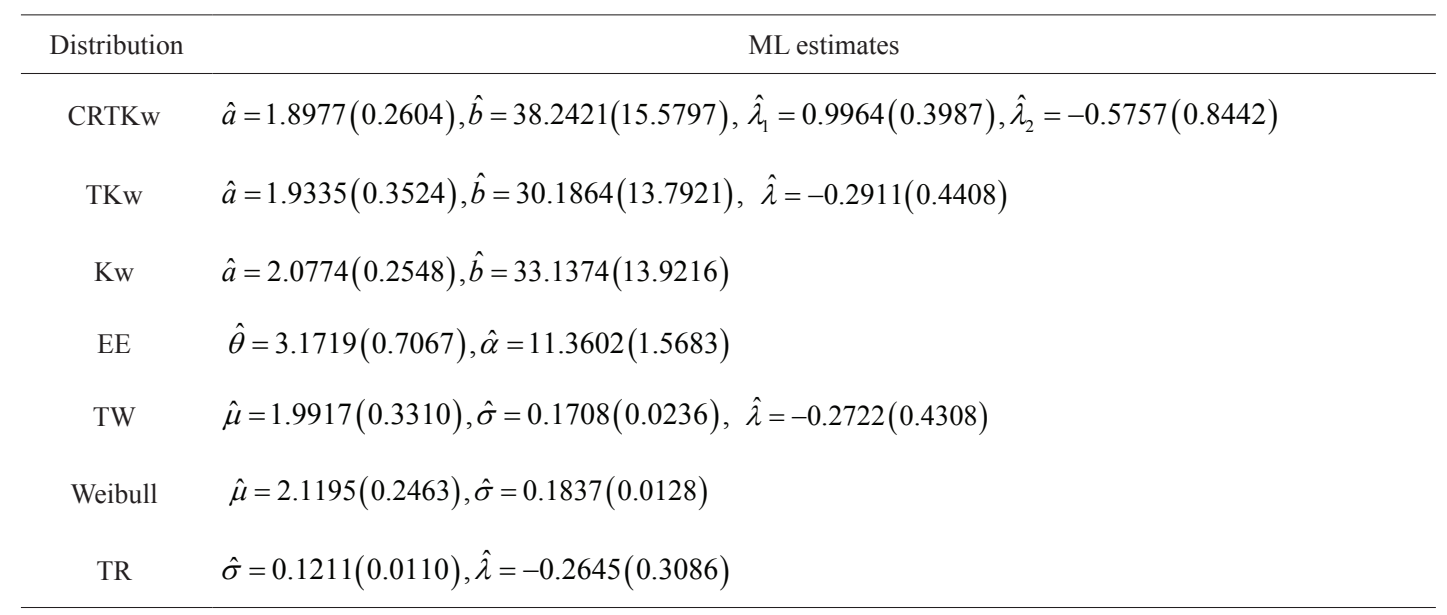

Table 6: Selection criteria statistics for operation and empirical data

\begin{tabular}{cccccccc}
\hline Distribution & $-2 \log$ & AIC & AICc & BIC & A* & K-S & p value \\
\hline CRTKw & $\mathbf{- 1 1 5 . 2 3 4}$ & -107.234 & -97.457 & -99.586 & $\mathbf{0 . 4 7 3}$ & 0.1139 & 0.5359 \\
TKw & -112.502 & -106.502 & -105.980 & -100.766 & 0.625 & 0.1052 & 0.6375 \\
Kw & -112.137 & -108.137 & -107.882 & -104.313 & 0.677 & 0.1103 & 0.5777 \\
TR & -112.117 & -108.117 & -107.862 & -104.293 & 2.004 & 0.1070 & 0.6159 \\
TW & -112.117 & -106.117 & -105.596 & -100.381 & 0.661 & 0.1070 & 0.6163 \\
Weibull & -111.783 & -107.783 & -107.528 & -103.959 & 0.708 & 0.1099 & 0.5815 \\
EE & -104.572 & -100.572 & -100.317 & -96.748 & 1.261 & 0.1653 & 0.1302 \\
\hline
\end{tabular}

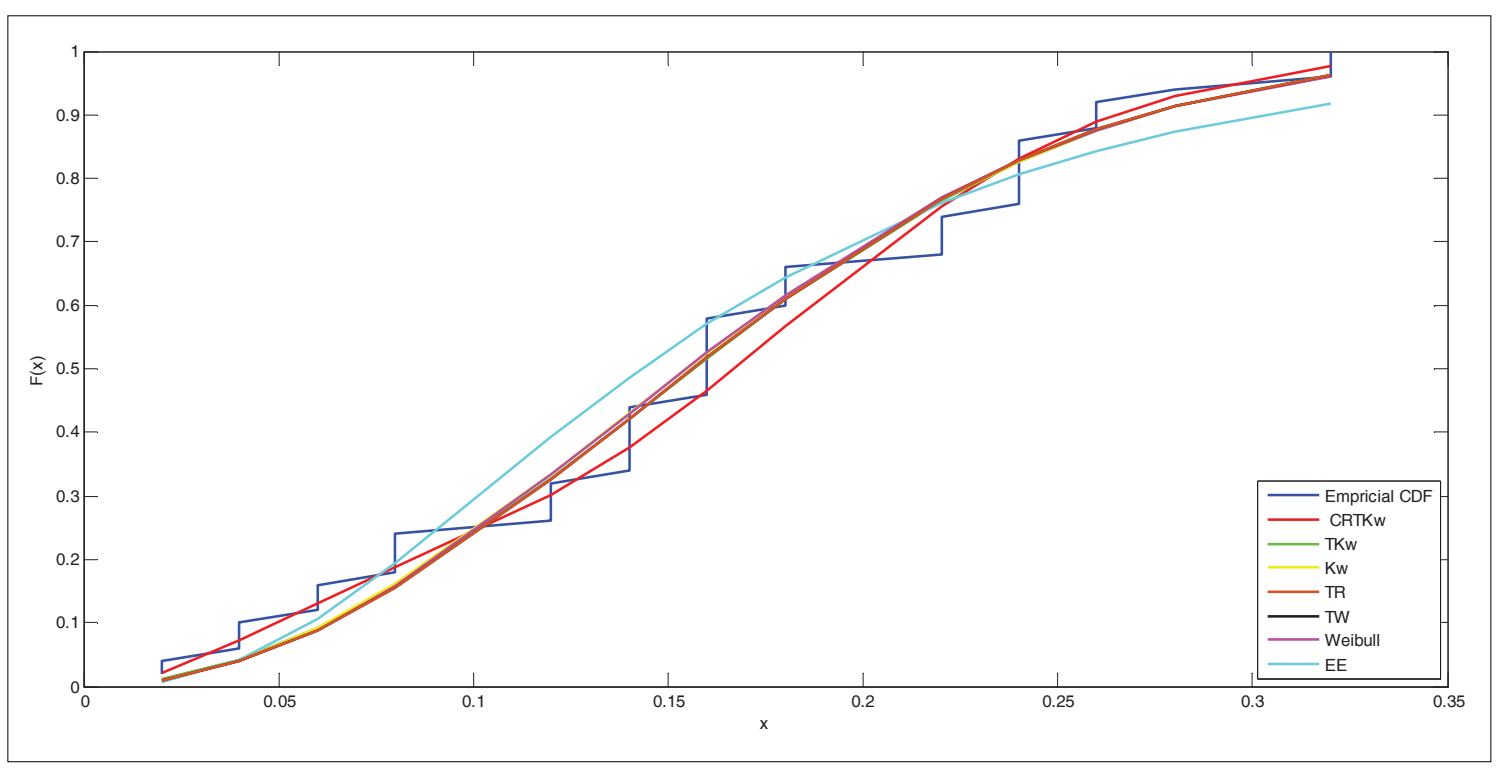

Figure 10: Empirical cdf and theoretical cdf for operation and empirical data 


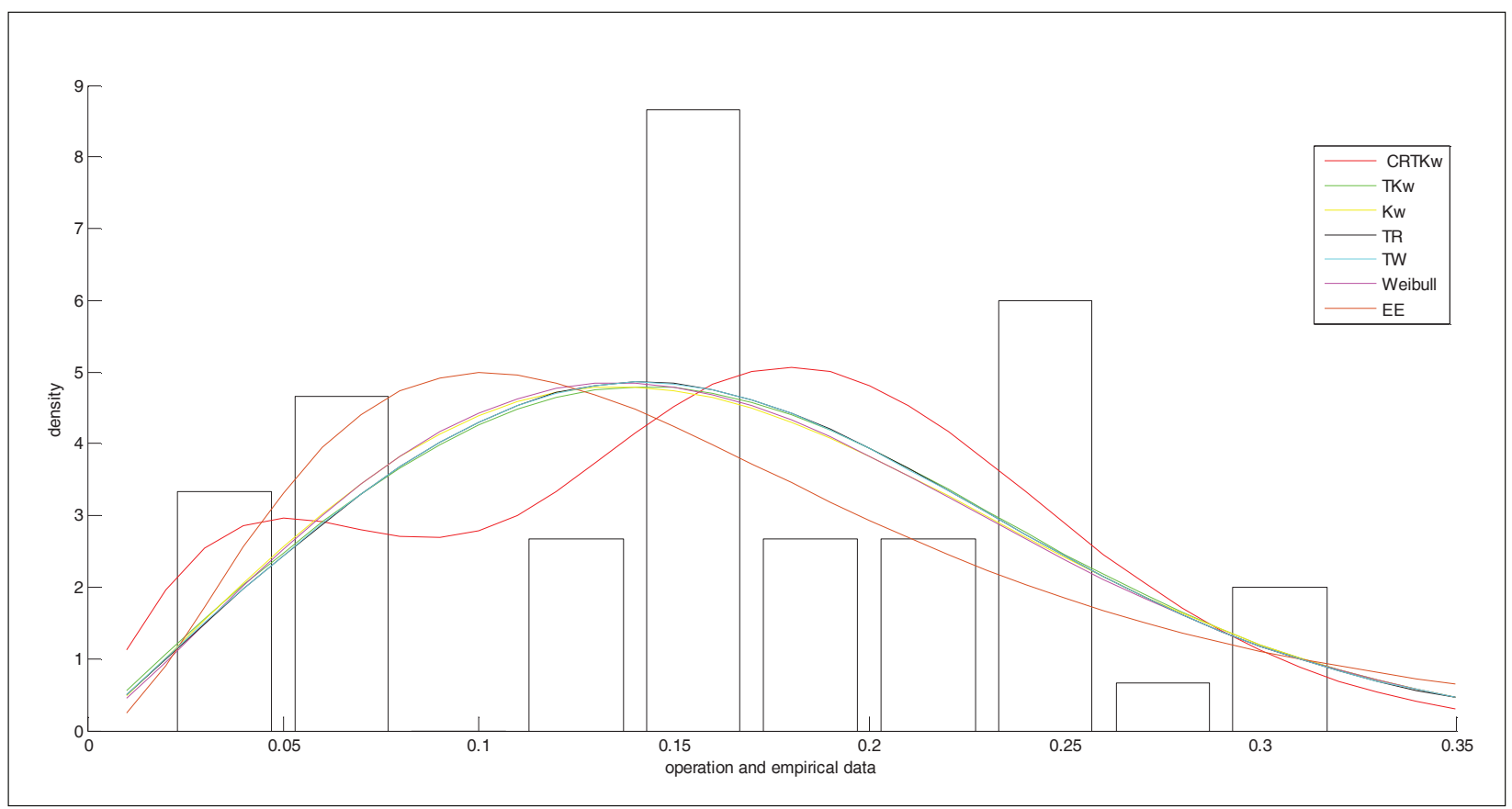

Figure 11: Fitted pdf for operation and empirical dataset

\section{CONCLUSION}

In this study, we have introduced a new model called cubic rank transmuted Kumaraswamy (CRTKw) distribution with parameters $\left(a, b, \lambda_{1}, \lambda_{2}\right)$ by using the cubic rank transmutation map as suggested by Granzotto et al. (2017). Some statistical properties of this new model are obtained. Further, the maximum likelihood estimator for unknown parameters of the CRTKw distribution is obtained. The performance of this estimator by means of a Monte-Carlo simulation study has been examined in terms of mean square error and bias.

In real data analysis, the dataset of Cordeiro and dos Santos Brito (2012a), and Brito (2009) has been used first. Table 3 shows that all of goodness-of-fit measures has the smallest value for CRTKw distribution. Therefore, the CRTKw distribution has a better fit than the other fitted distributions. Secondly, the operation and empirical dataset obtained by Dasgupta (2011) is considered. It can be seen that CRTKw distribution is the distribution that best fits among all fitted distributions according to Anderson-Darling goodness-of-fit statistics $\left(\mathrm{A}^{*}\right)$ and $-2 \times \log$-likelihood values in Table 6 . Moreover, $\mathrm{Kw}$ distribution has the best fit in terms of AIC, AICc and BIC criteria. As a result, CRTKw distribution can be used for analysis of these two datasets.
This new distribution called the CRTKw distribution can be used to model skewed data and the datasets having increasing and bathtube failure rates.

\section{REFERENCES}

Afify A.Z., Cordeiro G.M., Yousof H.M., Alzaatreh A. \& Nofal Z.M. (2016). The Kumaraswamy transmuted-G family of distributions: properties and applications. Journal of Data Science 14(2): 245 - 270.

Akdoğan Y., Kuş C., Asgharzadeh A., Kınacı İ. \& Sharafi F. (2016). Uniform-geometric distribution. Journal of Statistical Computation and Simulation 86(9): $1754-1770$.

DOI: https://doi.org/10.1080/00949655.2015.1081907

Al-Babtain A., Fattah A.A., Ahmed A.H.N. \& Merovci F. (2017). The Kumaraswamy-transmuted exponentiated modified Weibull distribution. Communications in Statistics-Simulation and Computation 46(5): 3812 - 3832.

Brito R.S. (2009). Estudo de Expansões Assintóticas, Avaliação Numérica de Momentos das Distribuições Beta Generalizadas, Aplicações em Modelos de Regressão e Análise Discriminante. Master's thesis, Universidade Federal Rural de Pernambuco.

Celik N. \& Guloksuz C.T. (2017). A new lifetime distribution (Nowy Rozkład Cyklu Życia). Eksploatacja I Niezawodnosc (Maintenance and Reliability) 19(4): $634-639$.

DOI: https://doi.org/10.17531/ein.2017.4.18 
Cordeiro G.M. \& de Castro M. (2011). A new family of generalized distributions. Journal of Statistical Computation and Simulation 81(7): 883 - 898. DOI: https://doi.org/10.1080/00949650903530745

Cordeiro G.M. \& dos Santos Brito R. (2012a). The beta power distribution. Brazilian Journal of Probability and Statistics 26(1): 88 - 112.

DOI: https://doi.org/10.1214/10-BJPS124

Cordeiro G.M., Nadarajah S. \& Ortega E.M.M. (2012b). The Kumaraswamy Gumbel distribution. Statistical Methods and Applications 21(2): 139 - 168.

DOI: https://doi.org/10.1007/s10260-011-0183-y

Cordeiro G.M., Ortega E.M.M. \& Nadarajah S. (2010). The Kumaraswamy Weibull distribution with application to failure data. Journal of the Franklin Institute 347(8): $1399-1429$. DOI: https://doi.org/10.1016/j.jfranklin.2010.06.010

Dasgupta R. (2011). On the distribution of burr with applications. Sankhya B 73(1): 1 - 19.

DOI: https://doi.org/10.1007/s13571-011-0015-y

Elgarhy M., Haq M. \& Ozel G. (2017). A new exponentiated extended family of distributions with applications. Gazi University Journal of Science 30(3): 101 - 115.

Ganji A., Ponnambalam K., Khalili D. \& Karamouz M. (2006). Grain yield reliability analysis with crop water demand uncertainty. Stochastic Environmental Research and Risk Assessment 20(4): $259-277$.

DOI: https://doi.org/10.1007/s00477-005-0020-7

Gradstein I.S. \& Ryzhik I.M. (1994). Tables of Integrals, Sums, Series and Products, $5^{\text {th }}$ Edition, Academic Press, Inc., Massachusetts, USA.

Granzotto D.C.T., Louzada F. \& Balakrishnan N. (2017) Cubic rank transmuted distributions: inferential issues and applications. Journal of Statistical Computation and Simulation 87(14): 2760 - 2778.

DOI: https://doi.org/10.1080/00949655.2017.1344239
Khan M.S., King R. \& Hudson I.L. (2016). Transmuted Kumaraswamy Distribution. Statistics in Transition New Series 17(2): $183-210$.

Korkmaz M.Ç. \& Genç A.İ. (2017). A new generalized twosided class of distributions with an emphasis on twosided generalized normal distribution. Communications in Statistics-Simulation and Computation 46(2): 1441 - 1460. DOI: https://doi.org/10.1080/03610918.2015.1005233

Kumaraswamy P. (1980). A generalized probability density function for double-bounded random processes. Journal of Hydrology 46(1 - 2): $79-88$.

Kuş C. (2007). A new lifetime distribution. Computational Statistics and Data Analysis 51(9): 4497 - 4509. DOI: https://doi.org/10.1016/j.csda.2006.07.017

Nofal Z.M., Afify A.Z., Yousof H.M., Granzotto D.C. \& Louzada F. (2016). Kumaraswamy transmuted exponentiated additive Weibull distribution. International Journal of Statistics and Probability 5(2): 78 - 99. DOI: https://doi.org/10.5539/ijsp.v5n2p78

Shaw W.T. \& Buckley I.R. (2009). The alchemy of probability distributions: beyond Gram-Charlier expansions, and a skew-kurtotic-normal distribution from a rank transmutation map. arXiv:0901.0434.

ul Haq M.A., Butt N.S., Usman R.M. \& Fattah A.A. (2016). Transmuted power function distribution. Gazi University Journal of Science 29(1): 177 - 185.

Usman R.M., ul Haq M.A. \& Talib J. (2017). Kumaraswamy half-logistic distribution: properties and applications. Journal of Statistics Applications and Probability 6: $597-609$.

DOI: https://doi.org/10.18576/jsap/060315

Yousof H.M., Alizadeh M., Jahanshahi S.M.A., Ramires T.G., Ghosh I. \& Hamedani G.G. (2017). The transmuted ToppLeone $\mathrm{G}$ family of distributions: theory, characterizations and applications. Journal of Data Science 15: 723 - 740. 